\title{
Antipodal Paraunitary Matrices and Their Application to OFDM Systems
}

\author{
See-May Phoong, Senior Member, IEEE, and Kai-Yen Chang
}

\begin{abstract}
Paraunitary (PU) matrices and filterbanks have played an important role in many applications. This paper studies a special class of PU matrices, called antipodal paraunitary (APU) matrices. APU matrices are PU matrices whose coefficient matrices consist of \pm 1 only. Several new methods will be introduced for the construction of APU matrices. In particular, a new method based on the butterfly structure that has a low cost implementation is proposed. Moreover, one application of APU matrices in precoded orthogonal frequency division multiplexing (OFDM) systems will be considered. By using an APU precoding matrix, the complexity will be very low, and experiments show that the use of APU matrices can greatly enhance the performance.
\end{abstract}

Index Terms-Antipodal paraunitary matrices, filterbanks, OFDM.

\section{INTRODUCTION}

$\mathbf{M}$ ULTIRATE systems and filterbanks (FBs) have played an important role in various areas of signal processing [1]-[3]. They have been successfully applied to source coding and compression, subband adaptive filtering, denoising, multicarrier communications, etc. Of particular interest is the class of paraunitary (PU) matrices and FBs. One attractive feature of these matrices is their energy preservation property that can avoid the noise or error amplification problem. In the past, the design and complete parameterization of PU matrices have been successfully derived [4], [5]. In this paper, we are going to study a special class of PU matrices and FBs, namely, antipodal paraunitary (APU) matrices and FBs. A PU matrix is said to be an APU matrix if all of its coefficient matrices have \pm 1 as their entries. For the special case of constant (memoryless) matrices, APU matrices reduce to the well-known Hadamard matrices. One of the attractive features of these matrices is its low implementational cost; only additions are needed.

APU matrices are closely related to the complementary sequences (or more commonly known as the Golay sequences) [6] and the $M$-shift cross-orthogonal sequences [7]-[10]. The applications of APU matrices and complementary sequences in areas of communications such as synchronous spread spectrum communications and code division multiple access (CDMA) systems have been explored in [11] and [12]. In the past, many construction methods for APU matrices have been proposed [7],

\footnotetext{
Manuscript received November 2, 2003; revised March 29, 2004. This work was supported by the National Science Council, Taiwan, R.O.C., under Grant NSC 93-2752-E-002-006-PAE and NSC 93-2213-E-002-103. The associate editor coordinating the review of this manuscript and approving it for publication was Prof. Tulay Adali.

The authors are with the Department of Electrical Engineering and the Graduate Institute of Communication Engineering, National Taiwan University, Taipei, Taiwan, R.O.C. (e-mail: smp@ @cc.ee.ntu.edu.tw).

Digital Object Identifier 10.1109/TSP.2005.843735
}

[9], [11], [13], [14]. In [13] and [14], it is shown that we can construct $2 \times 2$ APU matrices by cascading $2 \times 2$ Hadamard matrices and diagonal matrices with delay elements. This method is extended for the construction of $M \times M$ APU matrices in [11]. In [7], it was shown that given a pair of complementary sequences, we can construct $2 \times 2$ APU matrices. Moreover, it was shown that the corresponding time-domain sequences of the APU matrices and their 2-shifts form an orthogonal basis for the class of finite energy signals. In [9], several methods are proposed for the construction of $M \times M$ APU matrices from APU matrices of smaller dimensions. Except for [11], most of these studies use the time domain approach, and there are relatively few systematic studies on the theory and construction methods of APU matrices.

In this paper, we apply the theory of PU matrices to the study of APU matrices. All the derivations will be done using a $z$-domain approach. This approach not only gives a compact description of the previous results but also enables us to generalize previous methods. In addition, several new construction methods are proposed. In particular, a new construction method based on the well-known butterfly structure is introduced. APU matrices constructed using the butterfly structure can be implemented efficiently and, hence, have very low cost. Existence issue of APU matrices will also be explored. Unlike PU matrices, there are some constraints on the degree, order, and dimension of APU matrices. We also consider one potential application of APU matrices. OFDM systems with APU precoding matrices are studied. By choosing APU matrices as the precoding matrix, we can have very low complexity. Moreover, we are able to average the noise variance in both the time and frequency domains, and this achieves time and frequency diversity. Both zero-forcing and MMSE receivers for the precoded OFDM systems will be derived. Simulation results show that precoded OFDM systems have a much better performance than the OFDM system.

The paper is organized as follows. In Section II, we first give some definitions and notations. Then, the connection between the orthogonal sequences and APU matrices will be reviewed in Section III. Some existing construction methods of APU matrices will be described in Section IV. A number of new construction methods will be introduced in Section V. The existence issue of APU matrices will be explored in Section VI. In Section VII, we study OFDM systems with APU precoding matrices. We will analyze the noise performance of these systems. Numerical experiments are provided in Section VIII to compare the performances of the precoded OFDM systems. Conclusions are given in Section IX. Parts of the results have been presented in conferences in [15] and [16]. 


\section{DEFINITIONS AND NOTATIONS}

In this section, we will introduce some definitions and notations. These preliminaries not only make this paper more self-contained but also facilitate the development of a $z$-domain framework later.

1) Antipodal Sequences and Polynomials: Sequences are denoted as lowercase letters with index $n$, such as $a(n)$. The discrete unit impulse is denoted by $\delta(n)$. It is equal to 1 when $n=0$ and 0 otherwise. All sequences studied in this paper are finite impulse response (FIR). A causal sequence $a(n)$ of length $N$ is said to be antipodal (AP) if $a(n)= \pm 1$ for $0 \leq n<N$ and 0 otherwise. The $z$-transforms of sequences are denoted by the corresponding uppercase letters, such as $A(z)=\sum_{n} a(n) z^{-n}$. The $z$-transform of an AP sequence will be called an AP polynomial.

2) All vectors and matrices are represented by boldfaced letters. The symbols $\mathbf{I}$ and $\mathbf{P}$ are reserved for the identity and permutation matrices, respectively. $\mathbf{A}^{T}$ and $\mathbf{A}^{H}$ denote the transpose and the transpose conjugate of $\mathbf{A}$, respectively. A constant matrix is said to be AP if all of its entries are \pm 1 .

3) A polynomial matrix will be denoted by $\mathbf{A}(z)$. A polynomial matrix $\mathbf{A}(z)=\sum_{n=0}^{N-1} \mathbf{A}_{n} z^{-n}$ is AP if $\mathbf{A}_{n}$ are AP for $n=0,1, \ldots, N-1$.

4) Order, Length, and Degree: Let $\mathbf{A}(z)=$ $\sum_{n=0}^{N-1} \mathbf{A}_{n} z^{-n}$ with both $\mathbf{A}_{0} \neq \mathbf{0}$ and $\mathbf{A}_{N-1} \neq \mathbf{0}$. Then the order and length of $\mathbf{A}(z)$ are, respectively, $N-1$ and $N$. Its degree is the minimum number of delays needed to implement $\mathbf{A}(z)$. For example, the order of $\mathbf{A}_{0}+\mathbf{A}_{1} z^{-1}$ is equal to 1, while its degree is equal to the rank of $\mathbf{A}_{1}$.

5) Tilde Notation: Given a polynomial matrix $\mathbf{A}(z)$, its tilde is defined [1] as

$$
\tilde{\mathbf{A}}(z)=\mathbf{A}^{H}\left(1 / z^{*}\right) .
$$

For a polynomial matrix $\mathbf{A}(z)$ with real coefficients, $\tilde{\mathbf{A}}(z)=\mathbf{A}^{T}\left(z^{-1}\right)$.

6) Cross Correlation: Given two sequences $a(n)$ and $b(n)$, their $k$ th cross correlation coefficient is given by

$$
r_{a b}(k)=\sum_{n} a(n) b^{*}(n-k) .
$$

Using the tilde notation, the z-transform of the crosscorrelation coefficients can be expressed as

$$
R_{a b}(z)=\sum_{k} r_{a b}(k) z^{-k}=A(z) \tilde{B}(z) .
$$

7) Kronecker Product of Matrices: Given two square polynomial matrices $\mathbf{A}(z)$ and $\mathbf{B}(z)$ with dimensions $M_{a}$ and $M_{b}$ respectively, their Kronecker product is defined as shown in the equation at the bottom of the page. Note that $\mathbf{A}(z) \otimes \mathbf{B}(z)$ is an $M_{a} M_{b} \times M_{a} M_{b}$ matrix. Moreover, if the orders of $\mathbf{A}(z)$ and $\mathbf{B}(z)$ are $N_{a}$ and $N_{b}$, respectively, then the order of $\mathbf{A}(z) \otimes \mathbf{B}(z)$ will be $N_{a}+N_{b}$. Note that when the matrices are constant matrices independent of $z$, then the above definition reduces to the conventional Kronecker product. One can verify that the tilde of $\mathbf{A}(z) \otimes \mathbf{B}(z)$ is equal to $\tilde{\mathbf{A}}(z) \otimes \tilde{\mathbf{B}}(z)$. Let the dimensions of the matrices $\mathbf{A}(z), \mathbf{B}(z), \mathbf{C}(z)$, and $\mathbf{D}(z)$ be so that all the matrix multiplications in the following expression are valid. Then, the product rule states that

$(\mathbf{A}(z) \otimes \mathbf{B}(z))(\mathbf{C}(z) \otimes \mathbf{D}(z))$

$$
=(\mathbf{A}(z) \mathbf{C}(z)) \otimes(\mathbf{B}(z) \mathbf{D}(z)) .
$$

8) Paraunitary and Normalized Paraunitary Matrices: An $M \times M$ polynomial matrix $\mathbf{A}(z)$ is said to be paraunitary (PU) [1] if

$$
\tilde{\mathbf{A}}(z) \mathbf{A}(z)=|c|^{2} \mathbf{I}_{M}
$$

for some nonzero constant $c$. When $|c|=1$, we say that $\mathbf{A}(z)$ is a normalized PU matrix. The above relation implies that $\mathbf{A}\left(e^{j \omega}\right)$ is unitary for all frequency $\omega$. It is well known that normalized PU matrices enjoy the energy-preservation property [1]. That is, if the input $\mathbf{x}(n)$ of a normalized PU matrix is a vector sequence of finite energy, the output vector $\mathbf{y}(n)$ satisfies

$$
\sum_{n} \mathbf{y}^{H}(n) \mathbf{y}(n)=\sum_{n} \mathbf{x}^{H}(n) \mathbf{x}(n)
$$

Using the product rule, one can show that given two PU matrices $\mathbf{A}(z)$ and $\mathbf{B}(z)$ (not necessarily of the same dimensions), their Kronecker product $\mathbf{A}(z) \otimes \mathbf{B}(z)$ is also PU.

9) Hadamard Matrices: An $M \times M$ Hadamard matrix, denoted by $\mathcal{H}_{M}$, is a constant AP matrix (independent of $z$ ) that satisfies $\mathcal{H}_{M}^{T} \mathcal{H}_{M}=M \mathbf{I}_{M}$. It was shown [17] that a necessary condition for the existence of a Hadamard matrix is that $M$ must be 2 or a multiple of 4 . Whether this is also a sufficient condition is still not known. Most of these Hadamard matrices have been successfully constructed; see [8] and references therein.

Antipodal PU Matrices: If a PU matrix $\mathbf{A}(z)$ is also antipodal, then it is called an antipodal paraunitary (APU)

$$
\mathbf{A}(z) \otimes \mathbf{B}(z)=\left(\begin{array}{cccc}
A_{00}(z) \mathbf{B}(z) & A_{01}(z) \mathbf{B}(z) & \cdots & A_{0, M-1}(z) \mathbf{B}(z) \\
A_{10}(z) \mathbf{B}(z) & A_{11}(z) \mathbf{B}(z) & \cdots & A_{1, M-1}(z) \mathbf{B}(z) \\
\vdots & \vdots & \ddots & \vdots \\
A_{M-1,0}(z) \mathbf{B}(z) & A_{M-1,1}(z) \mathbf{B}(z) & \cdots & A_{M-1, M-1}(z) \mathbf{B}(z)
\end{array}\right)
$$


matrix. It is not difficult to verify that if an $M$ by $M$ matrix $\mathbf{A}(z)=\sum_{n=0}^{N-1} \mathbf{A}_{n} z^{-n}$ is APU, then it satisfies $\tilde{\mathbf{A}}(z) \mathbf{A}(z)=\mathbf{A}(z) \tilde{\mathbf{A}}(z)=M N \mathbf{I}_{M}$, and its inverse $\tilde{\mathbf{A}}(z)$ is also APU.

\section{CRoss-Orthogonal Sequences AND APU Matrices}

In the following, we will first define three types of sequences that are widely used in communications and then describe the connection between APU matrices and these sequences.

1) A set of $M$ antipodal sequences of length $N, a_{i}(n)$, for $i=0,1, \ldots, M-1$, is said to be complementary [9] if

$$
\sum_{i=0}^{M-1} r_{a_{i} a_{i}}(k)=M N \delta(k) .
$$

When $M=2$, the complementary sequences are also known as the Golay codes [6].

2) An antipodal sequence of length $N, b(n)$ is called an $M$-shift orthogonal sequence [7], [10] if

$$
r_{b b}(l M)=N \delta(l)
$$

$M$-shift orthogonal sequences are closely related to complementary sequences. To see this, let $a_{i}(n)=$ $b(M n+i)$. Then, one can verify that $a_{i}(n)$ are complementary if and only if $b(n)$ is $M$-shift orthogonal.

3) A set of antipodal sequences $b_{i}(n), i=0,1, \ldots, K-1$ is called a set of $M$-shift cross-orthogonal sequences [10] if i) each sequence $b_{i}(n)$ is an $M$-shift orthogonal sequences and ii) any pair of distinct sequences $b_{i}(n)$ and $b_{j}(n)$ satisfies $r_{b_{i}} b_{j}(l M)=0$ for all integers $l$.

These sequences have been studied extensively in [6], [7], [9], and [10], and they have found many applications in communications [10]-[12].

1) APU Matrices and M-Shift Orthogonal Sequences: The sequences defined above are closely related to the APU matrices. From (2), we see that if an $M$ by $M$ matrix $\mathbf{E}(z)$ of length $N$ is APU, then its entries $E_{k i}(z)$ satisfy

$$
\sum_{i=0}^{M-1} E_{k i}(z) \tilde{E}_{k i}(z)=M N, \quad \text { for } k=0,1, \ldots, M-1 .
$$

Note that the above expression is nothing but the z-domain formulation of (4). Thus, the $M$ sequences $e_{k i}(n)$ for $i=0,1, \ldots, M-1$, on the $k$ th row of an APU matrix $\mathbf{E}(z)$, are complementary for $k=0,1, \ldots, M-1$. Moreover, if we use $\mathbf{E}(z)$ as the polyphase matrix of the analysis filterbank [1], then the analysis impulse responses $h_{k}(n)$ satisfy

$$
\sum_{n} h_{j}(n) h_{k}(n+M i)=M N \delta(i) \delta(k-j)
$$

for $0 \leq k<M$. In other words, the impulse response $h_{k}(n)$ is an $M$-shift orthogonal sequence, and any two analysis filters form a pair of $M$-shift cross-orthogonal sequences. Therefore, the study and construction of a set of $M$-shift cross-orthogonal sequences are identical to those of the APU matrices. In the following sections, we will see that FB theory not only greatly simplifies the derivations of many existing results but also leads us to some new construction methods for orthogonal sequences.

\section{Z-DOMAIN APPROACH TO EXISTING CONSTRUCTION METHODS OF APU MATRICES}

In the literature, a number of construction methods for APU matrices have been proposed. The earliest report dated back to 1969 when Taki et al. [7] successfully constructed 2 by 2 APU matrices using complementary sequences [6]. The $M$ by $M$ case was studied in [9] and [11]. In this section, we will review some existing construction methods of APU matrices. These results are mostly derived in the time domain in the original works. We will describe them using the $z$-domain framework. The $z$-transform approach not only greatly simplifies the derivation but also allows us to make comparisons with the proposed methods later on.

Before we illustrate the construction of APU matrices, we will state a simple result that is useful for the understanding of the following construction method. Let

$$
A(z)=\sum_{n=0}^{N_{a}-1} a_{n} z^{-n}, \quad \text { and } \quad B(z)=\sum_{n=0}^{N_{b}-1} b_{n} z^{-n}
$$

be two AP polynomials. Then, it is clear that the following three polynomials

$$
\begin{aligned}
& A(z) B\left(z^{N_{a}}\right), \quad A\left(z^{N_{b}}\right) B(z), \quad A(z)+z^{-N_{a}} B(z), \quad \text { and } \\
& z^{-N_{b}} A(z)+B(z)
\end{aligned}
$$

are AP polynomials. The lengths of the first two and the last two polynomials are $N_{a} N_{b}$ and $N_{a}+N_{b}$, respectively.

\section{A. 2 by 2 APU Matrices}

APU matrices that are 2 by 2 are closely related to complementary sequences or, more commonly, Golay sequences [6], [7]. Recall from the previous section that a pair of AP sequences $a(n)$ and $b(n)$ of length $N$ are complementary if they satisfy

$$
A(z) \tilde{A}(z)+B(z) \tilde{B}(z)=2 N .
$$

It is well known [1] that given a pair of polynomials satisfying (6), we can form a two-channel PU filterbank by choosing the two analysis filters as

$$
H_{0}(z)=A\left(z^{2}\right)+z^{-1} B\left(z^{2}\right), \quad H_{1}(z)=z^{-2 N+1} \tilde{H}_{0}(-z) .
$$

Such a construction has been derived by Taki et al. in [7]. It was shown therein that even shifts of $h_{0}(n)$ and $h_{1}(n)$ form an orthogonal basis for the class of finite energy signals. Let $\mathbf{E}(z)$ be the analysis polyphase matrix

$$
\mathbf{E}(z)=\left(\begin{array}{cc}
A(z) & B(z) \\
-z^{-N+1} \tilde{B}(z) & z^{-N+1} \tilde{A}(z)
\end{array}\right) .
$$

Using (6), it is straightforward to verify that $\tilde{\mathbf{E}}(z) \mathbf{E}(z)=2 N \mathbf{I}$. The matrix $\mathbf{E}(z)$ is APU. Hence, every pair of Golay sequences generates a 2 by 2 APU matrix. Golay sequences with length 
$N=2^{a} 10^{b} 26^{c}$ (where $a, b, c$ are integers) have been successfully constructed in [6]. No Golay sequence of other lengths has been found thus far.

Another construction method for 2 by 2 APU matrices with length equal to a power of two was discovered independently by Shapiro [13] and Rudin [14]. Their algorithm is defined recursively as follows. Let $\mathbf{E}_{0}(z)=\mathcal{H}_{2}$ : the $2 \times 2$ Hadamard matrix. For $i \geq 1$, let $\mathbf{E}_{i}(z)$ be recursively defined as

$$
\mathbf{E}_{i}(z)=\mathcal{H}_{2} \Lambda\left(z^{2^{i-1}}\right) \mathbf{E}_{i-1}(z)
$$

where the diagonal matrix $\boldsymbol{\Lambda}(z)$ is

$$
\boldsymbol{\Lambda}(z)=\left(\begin{array}{cc}
1 & 0 \\
0 & z^{-1}
\end{array}\right)
$$

As $\mathcal{H}_{2}, \boldsymbol{\Lambda}\left(z^{2^{i-1}}\right)$, and $\mathbf{E}_{i-1}(z)$ are PU matrices, so is their product $\mathbf{E}_{i}(z)$ [1]. It is not difficult to verify that $\mathbf{E}_{i}(z)$ are AP for all $i$. Therefore, $\mathbf{E}_{i}(z)$ are APU matrices with length equal to $2^{i}$.

\section{B. $M \times M A P U$ Matrices}

The idea of Golay sequences was generalized to $M$ complementary sequences in [9]. Unlike the two-channel case, there is no known method to construct an $M$-channel APU-FB from a set of $M$ complementary sequences. Thus, the construction methods for $M$ complementary sequences were not helpful in generating $M$ by $M$ APU matrices. Nevertheless, there are algorithms that can generate APU matrices from APU matrices of smaller dimensions or lower orders. In the following, we will introduce four known methods.

Let $\mathbf{E}_{0}(z)$ be a $2 \times 2$ APU matrix of length $N$. Thus, $\tilde{\mathbf{E}}_{0}(z) \mathbf{E}_{0}(z)=2 N \mathbf{I}_{2}$. Using this $2 \times 2$ matrix as a starting point, we can recursively generate two different sets of APU matrices using the following interleaving and concatenating methods.

1) Tseng's Interleaving Method [9]: For $i \geq 1$, let $\mathbf{E}_{i}(z)$ be the $2^{i+1} \times 2^{i+1}$ matrix defined by (8), shown at the bottom of the page.

2) Tseng's Concatenating Method [9]: For $i \geq 1$, let $\mathbf{E}_{i}(z)$ be the $2^{i+1} \times 2^{i+1}$ matrix defined by (9), shown at the bottom of the page. One can verify by direct multiplication that $\tilde{\mathbf{E}}_{i}(z) \mathbf{E}_{i}(z)=4^{i} 2 N \mathbf{I}_{2^{i+1}}$. Hence, $\mathbf{E}_{i}(z)$ defined in the above two equations are PU matrices. Moreover, using (5), it is not difficult to see that $\mathbf{E}_{i}(z)$ are AP of length $2^{i} N$. Therefore, the interleaving and concatenating methods generate APU matrices. One can generalize the two methods by choosing
$\mathbf{E}_{0}(z)$ as any $M$ by $M$ APU matrix with length $N$. It is not difficult to show that in this case, the two methods will generate $2^{i} M$ by $2^{i} M$ APU matrices $\mathbf{E}_{i}(z)$ with length $2^{i} N$.

3) Tseng's Kronecker Product Method [9]': Let $\mathbf{E}(z)$ be an $M_{1}$ by $M_{1}$ APU matrix of length $N$. Let $M_{2}$ be an integer for which an $M_{2}$ by $M_{2}$ Hadamard matrix exists. Consider the following $M_{1} M_{2}$ by $M_{1} M_{2}$ matrix

$$
\mathcal{H}_{M_{2}} \otimes \mathbf{E}(z)
$$

Recall that the Kronecker product of two PU matrices is also PU. The AP property of the above matrix can be easily verified using the definition of Kronecker product. Thus, the matrix defined in (10) is an APU matrix of length $N$.

4) Wornell's Method: In [11], Wornell generalized the Shapiro-Rudin construction method to the $M$-channel case. Let $M$ be an integer for which $M \times M$ Hadamard matrix exists. Let $\boldsymbol{\Lambda}(z)$ be the diagonal matrix:

$$
\boldsymbol{\Lambda}(z)=\left(\begin{array}{cccc}
1 & 0 & \cdots & 0 \\
0 & z^{-1} & \cdots & 0 \\
\vdots & \vdots & \ddots & \vdots \\
0 & 0 & \cdots & z^{-M+1}
\end{array}\right)
$$

Let $\mathbf{E}_{0}(z)=\mathcal{H}_{M}$. Then, the algorithm can be described as [11]

$$
\mathbf{E}_{i}(z)=\mathcal{H}_{M} \boldsymbol{\Lambda}\left(z^{M^{i-1}}\right) \mathbf{E}_{i-1}(z)
$$

for $i \geq 1$. It is not difficult to verify that the above algorithm generates APU matrices of length $M^{i}$.

\section{New Construction Methods for APU Matrices}

In the following, we present three new construction methods for APU matrices. The first two methods are simple generalizations of Tseng's Kronecker product method and the Agayan-Sarukhanyan theorem [8]. The last construction method is based on the butterfly structure.

1) Generalized Kronecker Product Method: Let $\mathbf{A}(z)$ and $\mathbf{B}(z)$ be APU matrices of dimensions $M_{a}$ and $M_{b}$, respectively. Let $N_{a}$ and $N_{b}$ be their respective lengths. Consider the following two matrices:

$$
\begin{aligned}
& \mathbf{A}\left(z^{N_{b}}\right) \otimes \mathbf{B}(z) \\
& \mathbf{A}(z) \otimes \mathbf{B}\left(z^{N_{a}}\right) .
\end{aligned}
$$

${ }^{1}$ Alhough it was described differently in [9], we have chosen to express the results in a Kronecker product form as it gives a far more compact expression.

$$
\mathbf{E}_{i}(z)=\left(\begin{array}{cc}
\mathbf{E}_{i-1}\left(z^{2}\right)+z^{-1} \mathbf{E}_{i-1}\left(z^{2}\right) & -\mathbf{E}_{i-1}\left(z^{2}\right)+z^{-1} \mathbf{E}_{i-1}\left(z^{2}\right) \\
-\mathbf{E}_{i-1}\left(z^{2}\right)+z^{-1} \mathbf{E}_{i-1}\left(z^{2}\right) & \mathbf{E}_{i-1}\left(z^{2}\right)+z^{-1} \mathbf{E}_{i-1}\left(z^{2}\right)
\end{array}\right)
$$

$$
\mathbf{E}_{i}(z)=\left(\begin{array}{cc}
\mathbf{E}_{i-1}(z)+z^{-2^{(i-1)} N} \mathbf{E}_{i-1}(z) & -\mathbf{E}_{i-1}(z)+z^{-2^{(i-1)} N} \mathbf{E}_{i-1}(z) \\
-\mathbf{E}_{i-1}(z)+z^{-2^{(i-1)} N} \mathbf{E}_{i-1}(z) & \mathbf{E}_{i-1}(z)+z^{-2^{(i-1)} N} \mathbf{E}_{i-1}(z)
\end{array}\right)
$$


Using the fact that the Kronecker product of two PU matrices is also PU, one can see that the above two matrices are PU. The AP property of these matrices follows directly from (5). Hence, the two formulas in (12) and (13) generate two APU matrices with length $N_{a} N_{b}$ and dimension $M_{a} M_{b}$. When $\mathbf{A}(z)=$ $\mathcal{H}_{M_{2}}$, (13) reduces to Tseng's Kronecker product method. The above two seemingly simple generalizations of Tseng's Kronecker product method also include Tseng's interleaving and concatenating methods as special cases. To see this, let

$$
\begin{aligned}
& \mathbf{A}(z)=\left(\begin{array}{cc}
1+z^{-1} & -1+z^{-1} \\
-1+z^{-1} & 1+z^{-1}
\end{array}\right), \quad \text { and } \\
& \mathbf{B}(z)=\mathbf{E}_{i-1}(z) .
\end{aligned}
$$

Then, one can verify that (12) and (13) reduce, respectively, to Tseng's interleaving method in (8) and Tseng's concatenating method in (9).

2) Generalized Agayan-Sarukhanyan (AS) Method: Using the multiplication theorem of Agayan-Sarukhanyan (AS) [8], it was shown that given two Hadamard matrices of dimensions $M_{a}$ and $M_{b}$, one can construct a Hadamard matrix of dimension $M_{a} M_{b} / 2$. We now show that the result can be generalized to the case of APU matrices. Let $\mathbf{A}(z)$ and $\mathbf{B}(z)$ be APU matrices of dimensions $M_{a}$ and $M_{b}$, respectively. Suppose that their lengths are $N_{a}$ and $N_{b}$, respectively. Consider the following partitions:

$$
\begin{aligned}
& \mathbf{A}(z)=\left(\begin{array}{ll}
\mathbf{A}_{00}(z) & \mathbf{A}_{01}(z) \\
\mathbf{A}_{10}(z) & \mathbf{A}_{11}(z)
\end{array}\right) \\
& \mathbf{B}(z)=\left(\begin{array}{ll}
\mathbf{B}_{00}(z) & \mathbf{B}_{01}(z) \\
\mathbf{B}_{10}(z) & \mathbf{B}_{11}(z)
\end{array}\right)
\end{aligned}
$$

where $\mathbf{A}_{i j}(z)$ and $\mathbf{B}_{i j}(z)$ are $M_{a} / 2 \times M_{a} / 2$ and $M_{b} / 2 \times$ $M_{b} / 2$ matrices, respectively. This partition is always possible as $M_{a}$ and $M_{b}$ are even (see next section for a proof). Form the following $\left(\left(M_{a} M_{b}\right) / 2\right) \times\left(\left(M_{a} M_{b}\right) / 2\right)$ matrix with length $\left(N_{a} N_{b}\right)$ :

$$
\mathbf{C}(z)=\left(\begin{array}{ll}
\mathbf{C}_{00}(z) & \mathbf{C}_{01}(z) \\
\mathbf{C}_{10}(z) & \mathbf{C}_{11}(z)
\end{array}\right)
$$

where the submatrices are given by

$$
\begin{aligned}
\mathbf{C}_{00}(z)= & \frac{1}{2}\left(\mathbf{A}_{00}\left(z^{N_{b}}\right)+\mathbf{A}_{01}\left(z^{N_{b}}\right)\right) \otimes \mathbf{B}_{00}(z) \\
& +\frac{1}{2}\left(\mathbf{A}_{00}\left(z^{N_{b}}\right)-\mathbf{A}_{01}\left(z^{N_{b}}\right)\right) \otimes \mathbf{B}_{10}(z), \\
\mathbf{C}_{01}(z)= & \frac{1}{2}\left(\mathbf{A}_{00}\left(z^{N_{b}}\right)+\mathbf{A}_{01}\right)\left(z^{N_{b}}\right) \otimes \mathbf{B}_{01}(z) \\
& +\frac{1}{2}\left(\mathbf{A}_{00}\left(z^{N_{b}}\right)-\mathbf{A}_{01}\left(z^{N_{b}}\right)\right) \otimes \mathbf{B}_{11}(z), \\
\mathbf{C}_{10}(z)= & \frac{1}{2}\left(\mathbf{A}_{10}\left(z^{N_{b}}\right)+\mathbf{A}_{11}\left(z^{N_{b}}\right)\right) \otimes \mathbf{B}_{00}(z) \\
& +\frac{1}{2}\left(\mathbf{A}_{10}\left(z^{N_{b}}\right)-\mathbf{A}_{11}\left(z^{N_{b}}\right)\right) \otimes \mathbf{B}_{10}(z), \\
\mathbf{C}_{11}(z)= & \frac{1}{2}\left(\mathbf{A}_{10}\left(z^{N_{b}}\right)+\mathbf{A}_{11}\left(z^{N_{b}}\right)\right) \otimes \mathbf{B}_{01}(z) \\
& +\frac{1}{2}\left(\mathbf{A}_{10}\left(z^{N_{b}}\right)-\mathbf{A}_{11}\left(z^{N_{b}}\right)\right) \otimes \mathbf{B}_{11}(z) .
\end{aligned}
$$

The matrix $\mathbf{C}(z)$, formed in such a manner, is called the AS multiplication $^{2}$ of $\mathbf{A}\left(z^{N_{b}}\right)$ and $\mathbf{B}(z)$, which are denoted as

$$
\mathbf{C}(z)=\mathbf{A}\left(z^{N_{b}}\right) \otimes_{A S} \mathbf{B}(z)
$$

It is proved in the Appendix that the matrix $\mathbf{C}(z)$ is an APU matrix. Similarly, one can show that $\mathbf{A}(z) \otimes_{A S} \mathbf{B}\left(z^{N_{a}}\right)$ is also an $\left(\left(M_{a} M_{b}\right) / 2\right) \times\left(\left(M_{a} M_{b}\right) / 2\right)$ APU matrix with length $\left(N_{a} N_{b}\right)$. Let $M$ be such that Hadamard matrices of dimension $M$ exist. Then, we can apply the generalized AS multiplication to generate $M$ by $M$ APU matrices of length $N=2^{a} 10^{b} 26^{c}$ (where $a, b, c$ are integers). To see this, let $\mathbf{A}(z)$ be a 2 by 2 APU matrix with length $N=2^{a} 10^{b} 26^{c}$. Such an APU matrix can be obtained from Golay sequences (see Section IV-A). Then, the following matrix

$$
\mathbf{A}(z) \otimes_{A S} \mathcal{H}_{M}
$$

is an $M$ by $M$ APU matrix of length $N$. Comparing our results with the Wornell's method in (11), we see that the matrices constructed using (11) have length equal to $M^{k}$, whereas our matrices have length equal to $2^{a} 10^{b} 26^{c}$.

3) Butterfly Structure Method: Let $M$ be an integer for which an $M \times M$ Hadamard matrix exists. Define the following two $M \times M$ matrices:

$$
\mathcal{B}_{M}=\mathbf{I}_{M / 2} \otimes\left(\begin{array}{cc}
1 & 1 \\
1 & -1
\end{array}\right), \quad \boldsymbol{\theta}(z)=\mathbf{I}_{M / 2} \otimes\left(\begin{array}{cc}
1 & 0 \\
0 & z^{-1}
\end{array}\right)
$$

Let $\mathbf{E}_{0}(z)$ and $\mathbf{E}_{0}^{\prime}(z)$ be an $M \times M$ Hadamard matrix $\mathcal{H}$. Then, the following iterative procedure generates two classes of APU matrices:

$$
\begin{aligned}
& \mathbf{E}_{i}(z)=\mathcal{B}_{M} \boldsymbol{\theta}\left(z^{2^{i-1}}\right) \mathbf{P}_{i-1} \mathbf{E}_{i-1}(z) \\
& \mathbf{E}_{i}^{\prime}(z)=\mathbf{E}_{i-1}^{\prime}(z) \mathbf{P}_{i-1} \boldsymbol{\theta}\left(z^{2^{i-1}}\right) \mathcal{B}_{M}
\end{aligned}
$$

where $\mathbf{P}_{i}$ are arbitrary $M \times M$ permutation matrices. Because of the delays in $\boldsymbol{\theta}\left(z^{2^{i-1}}\right)$, the AP property is preserved. Moreover, $\mathbf{E}_{i}(z)$ is PU because it is a product of PU matrices. Hence, $\mathbf{E}_{i}(z)$ is APU, and its length is $2^{i}$. Similarly, one can show that $\mathbf{E}_{i}^{\prime}(z)$ is also APU with length $2^{i}$. For example, Fig. 1 shows $\mathbf{E}_{i}(z)$ for $i=2$ and $M=8$. Note that the butterfly structure has the additional advantage of low complexity. Its computational cost for adding one stage is $M$ additions. When $M$ is a power of two, the $M \times M$ Hadamard matrix $\mathcal{H}$ can also be realized using the butterfly structure [19]. This gives rise to an efficient implementation of APU matrices. Given an $M \times M$ APU matrix of length $N$, roughly $M^{2} N$ additions are needed in direct implementation. If both $M$ and $N$ are powers of 2 and the APU matrix is constructed using the butterfly structure, then only $M \log _{2}(M N)$ additions are needed.

4) Connection Between the Butterfly Structure Method and Wornell's Method [11]: When the number of channels $M$ is a power of two, we can show that the butterfly structure method

\footnotetext{
${ }^{2}$ The original multiplication is defined for constant matrices independent of $z$, and it is applied to the construction of Hadamard matrices [8].
} 


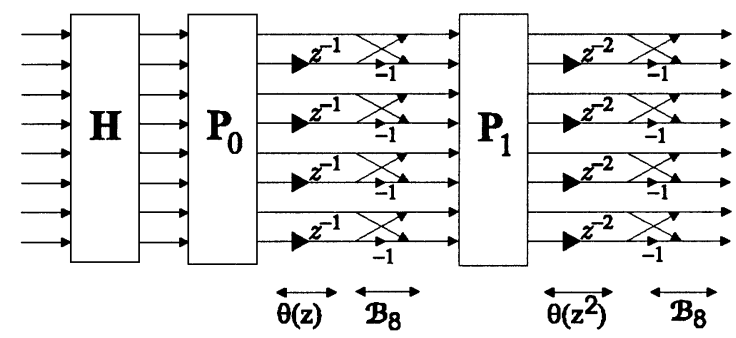

Fig. 1. An 8 by 8 APU matrix with length 4 constructed using the butterfly method.

(a)
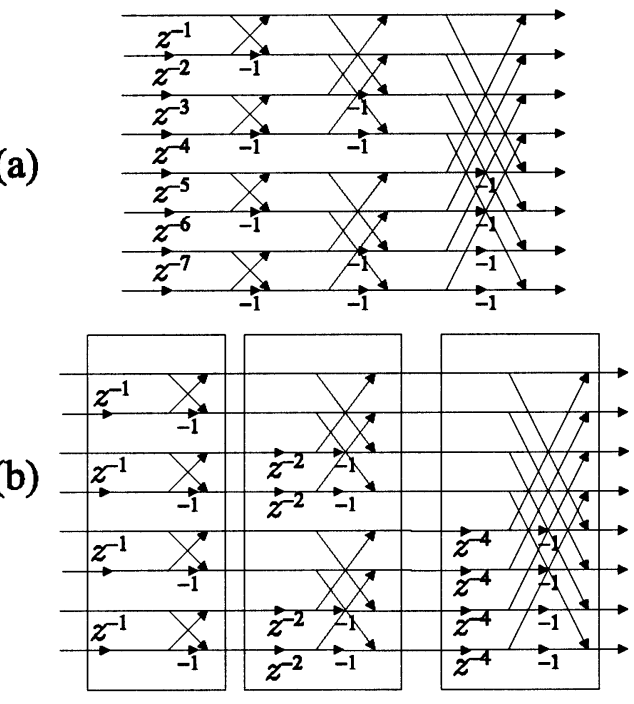

Fig. 2. (a) Efficient implementation of the 8 by 8 Hadamard matrix $\mathcal{H}_{8} \Lambda(z)$. (b) Equivalent system.

includes Wornell's method [11] as a special case. We demonstrate this for the case of $M=8$. To do this, we need to show that $\mathcal{H}_{8} \boldsymbol{\Lambda}(z)$ in (11) can be expressed a product of matrices of the form $\mathcal{B}_{8} \boldsymbol{\theta}(z) \mathbf{P}$, as in (16). It is well known [19] that the $8 \times 8$ Hadamard matrix can be implemented efficiently using butterflies. Using the butterfly structure, we can implement $\mathcal{H}_{8} \boldsymbol{\Lambda}(z)$ as Fig. 2(a). After moving some delay elements to the right of the butterflies, we can redraw Fig. 2(a) as Fig. 2(b). Note that the three stages (indicated by the dotted boxes) in Fig. 2(b) can be, respectively, described as $\mathcal{B}_{8} \boldsymbol{\theta}(z) \mathbf{P}_{0}, \mathcal{B}_{8} \boldsymbol{\theta}\left(z^{2}\right) \mathbf{P}_{1}$ and $\mathcal{B}_{8} \boldsymbol{\theta}\left(z^{4}\right) \mathbf{P}_{2}$ by choosing the permutation matrices $\mathbf{P}_{i}$ properly.

We summarize the different methods to obtain APU matrices in Table I. The possible dimensions $M$ and lengths $N$ of APU matrices generated from different methods are listed. The interleaving and concatenating methods, the generalized Kronecker product method, and the generalized AS method construct APU matrices from APU matrices of smaller dimensions. In each iteration, both the length and dimension of resulting APU matrices might increase. On the other hand, the Shapiro and Rudin method, the Wornell method, and the butterfly method can be used for APU matrices of a fixed dimension. Only the length of APU matrices increases in each iteration.

\section{5) Comments:}

1) Note that pre- or post-multiplying an APU matrix with either a permutation matrix or a diagonal matrix with all diagonal entries equal to \pm 1 generates another APU matrix.
TABLE I

SuMMARY OF DifFERENT METHODS TO GENERATE APU MATRICES With DimENSION $M$ AND LENGTH $N$. THE NUMBERS $M_{i}$ AND $N_{i}$ ARE INTEGERS FOR WHich APU MATRICES OF DIMENSIONS $M_{i}$ AND LENGTH $N_{i}$ EXIST

\begin{tabular}{l|c|c}
\hline & Dimension $M$ & Length $N$ \\
\hline method by Taki et al & 2 & $2^{a} 10^{b} 26^{c}$ \\
\hline Shaipro and Rudin method & 2 & $2^{a}$ \\
\hline interleaving and concatenating method & $2^{a}$ & $2^{a} N_{1}$ \\
\hline generalized Kronecker product method & $M_{1} M_{2}$ & $N_{1} N_{2}$ \\
\hline Wornell method & 2 or $4 a$ & $M^{b}$ \\
\hline generalized AS method & $M_{1} M_{2} / 2$ & $N_{1} N_{2}$ \\
\hline butterfly method & 2 or $4 a$ & $2^{b}$ \\
\hline
\end{tabular}

2) Except for the generalized AS multiplication, all the construction methods in the previous two sections can be modified so that they can be used to generate complex PU matrices with unit-magnitude coefficients. For example, if the Hadamard matrix $\mathcal{H}$ in (10) or (11) is replaced by a unitary matrix with unit-magnitude entries (the discrete Fourier transform (DFT) matrix is a matrix with such a property), then (10) or (11) will generate complex PU matrices with unit magnitude coefficients. For any integer $M$, we know that an $M$ by $M$ DFT matrix exists. Hence, we can generate $M$ by $M$ complex PU matrices with unit-magnitude coefficients for any $M$.

6) APU Matrices and Block Circulant Hadamard Matrices: Let $\mathbf{A}(z)$ be an $M \times M$ APU matrix with length $N$. That is, $\mathbf{A}(z)=\sum_{k=0}^{N-1} \mathbf{A}_{k} z^{-k}$. Then, using the time domain expression of $\tilde{\mathbf{A}}(z) \mathbf{A}(z)=M N \mathbf{I}$, one can verify by direct multiplication that the following $M N \times M N$ matrix is a Hadamard matrix:

$$
\left(\begin{array}{cccc}
\mathbf{A}_{0} & \mathbf{A}_{1} & \cdots & \mathbf{A}_{N-1} \\
\mathbf{A}_{N-1} & \mathbf{A}_{0} & \cdots & \mathbf{A}_{N-2} \\
\vdots & \vdots & \ddots & \vdots \\
\mathbf{A}_{1} & \mathbf{A}_{2} & \cdots & \mathbf{A}_{0}
\end{array}\right)
$$

The above Hadamard matrix has a block circulant structure. Thus, given any APU matrix, we can construct a block circulant Hadamard matrix.

7) Rectangular APU Matrices: One can extend the definition of APU matrices to include rectangular matrices. A $P \times M$ matrix $\mathbf{E}(z)=\sum_{n=0}^{N-1} \mathbf{E}_{n} z^{-n}$ is APU if all of entries of $\mathbf{E}_{i}$ are \pm 1 , and it satisfies $\tilde{\mathbf{E}}(z) \mathbf{E}(z)=P N \mathbf{I}_{M}$. Clearly, we have $P \geq M$. It is not difficult to verify that we can obtain rectangular APU matrices by deleting some columns of square APU matrices. Many previously described methods can be easily extended to derive rectangular APU matrices. For example, if we take $E_{0}(z)$ to be a rectangular submatrix formed by deleting some columns of a Hadamard matrix, then the interleaving and concatenating methods, the Wornell method, and the butterfly method will generate rectangular APU matrices.

\section{EXISTENCE IsSUES OF APU MATRICES}

It is known that Hadamard matrices exist only when the dimension is 2 or a multiple of 4 [17]. Whether this is also a sufficient condition is still unknown, but so far, no counter example has been found. In this section, we will study similar issues for 
APU matrices. We will first study the case of first-order APU matrices. Then, results for the more general case will be discussed.

Consider the $M \times M$ first-order APU matrix:

$$
\mathbf{A}(z)=\mathbf{A}_{0}+\mathbf{A}_{1} z^{-1} .
$$

Such a matrix is also known as a lapped transform. Applying the PU conditions in (2) to the matrix $\mathbf{A}(z)$, we can obtain the following four conditions:

$$
\begin{aligned}
\mathbf{A}_{0}^{T} \mathbf{A}_{1} & =\mathbf{0} \\
\mathbf{A}_{0} \mathbf{A}_{1}^{T} & =\mathbf{0} \\
\mathbf{A}_{0}^{T} \mathbf{A}_{0}+\mathbf{A}_{1}^{T} \mathbf{A}_{1} & =2 M \mathbf{I}_{M} \\
\mathbf{A}_{0} \mathbf{A}_{0}^{T}+\mathbf{A}_{1} \mathbf{A}_{1}^{T} & =2 M \mathbf{I}_{M} .
\end{aligned}
$$

As both $\mathbf{A}_{0}$ and $\mathbf{A}_{1}$ are AP matrices, one can immediately conclude from (19) or (20) that the dimension $M$ is even. From FB theory, it is well known [1] that all PU matrices can be minimally realized as a cascade of simple degree-one PU matrices. For the case of APU matrices, such simple degree-one APU building blocks do not exist (except for the special case of $M=2$ ). This is a direct consequence of the following theorem.

Theorem 1: Let $\mathbf{A}(z)=\mathbf{A}_{0}+\mathbf{A}_{1} z^{-1}$ be an $M \times M$ APU matrix. Then, $\operatorname{rank}\left(\mathbf{A}_{0}\right)=\operatorname{rank}\left(\mathbf{A}_{1}\right)=M / 2$.

Proof: Let $\operatorname{rank}\left(\mathbf{A}_{1}\right)=\rho$. From the factorization theorem of PU matrices [1], we know that

$$
\mathbf{A}(z)=\mathbf{A}(1)\left[\mathbf{I}_{M}-\mathbf{V}_{\rho} \mathbf{V}_{\rho}^{\dagger}+\mathbf{V}_{\rho} \mathbf{V}_{\rho}^{\dagger} z^{-1}\right]
$$

for some $M \times \rho$ matrix $\mathbf{V}_{\rho}$ satisfying $\mathbf{V}_{\rho}^{\dagger} \mathbf{V}_{\rho}=\mathbf{I}_{\rho}$. By direct substitution, one can verify that the matrix $\mathbf{A}(1)=\mathbf{A}_{0}+\mathbf{A}_{1}$. Using (19) and (21), we get $\mathbf{A}(1)^{T} \mathbf{A}(1)=2 M \mathbf{I}$. Multiplying both sides of (23) by $\mathbf{A}(1)^{T}$ and comparing the coefficient of $z^{-1}$, we obtain

$$
\left(\mathbf{A}_{0}+\mathbf{A}_{1}\right)^{T} \mathbf{A}_{1}=2 M \mathbf{V}_{\rho} \mathbf{V}_{\rho}^{\dagger}
$$

Since $\mathbf{A}_{0}^{T} \mathbf{A}_{1}=\mathbf{0}$, we have

$$
\operatorname{trace}\left(\mathbf{A}_{1}^{T} \mathbf{A}_{1}\right)=\operatorname{trace}\left(2 M \mathbf{V}_{\rho} \mathbf{V}_{\rho}^{\dagger}\right)=2 M \operatorname{trace}\left(\mathbf{V}_{\rho}^{\dagger} \mathbf{V}_{\rho}\right) .
$$

As trace $\left(\mathbf{A}_{1}^{T} \mathbf{A}_{1}\right)=M^{2}$ and $\mathbf{V}_{\rho}^{\dagger} \mathbf{V}_{\rho}=\mathbf{I}_{\rho}$, we get $M^{2}=2 M \rho$, which implies $\rho=M / 2$. From (23), we get $\operatorname{rank}\left(\mathbf{A}_{0}\right)=M-$ $\rho=M / 2$.

From the above theorem, we see that the degree of an $M \times M$ first-order APU matrix is $M / 2$. Thus, except for $M=2$, we cannot have a degree-one APU building block.

Although the existence issue of Hadamard matrices has been studied extensively (see [8] and references therein), there are essentially no reports on the existence issue of the APU matrices. From Wornell's method [11] and the butterfly method, we know that we can construct APU matrices from Hadamard matrices of the same dimension. Hence, we can immediately conclude that if $M$ is 2 or a multiple of 4 , APU matrices of higher order exist. However, since APU matrices are more general than Hadamard matrices in the sense that they have more free parameters, whether this is a necessary condition for the existence of APU matrices is still unknown. However, we are able to obtain a number of results listed below. Let $\mathbf{A}(z)=$ $\mathbf{A}_{0}+\mathbf{A}_{1} z^{-1}+\cdots+\mathbf{A}_{N-1} z^{-N+1}$ be an $M \times M$ APU matrix. Then, we have the following.

- The number $M$ is even. This can be verified from the facts that $\mathbf{A}_{0}$ and $\mathbf{A}_{N-1}$ are AP matrices satisfying $\mathbf{A}_{0}^{T} \mathbf{A}_{N-1}=\mathbf{0}$.

- $\quad$ If $N \neq 1$ is odd, then $M$ is a multiple of 4 . To see this, recall from (17) that we can have an $M N \times M N$ Hadamard matrix from $\mathbf{A}(z)$. As Hadamard matrices exist only for dimension equal to 2 or a multiple of 4 , if $N$ is an odd integer other than 1, then $M$ has to be a multiple of 4 .

- $\quad$ There does not exist any $2 \times 2$ APU matrix with odd $N \geq 3$. Otherwise, we could have constructed $2 N \times$ $2 N$ Hadamard matrices using (17), which is a contradiction.

There are a number of open problems. For example, it is still unclear if there exist APU matrices with odd length $\geq 3$. All the above construction methods generate APU matrices of even length only. In addition, we do not know if there are APU matrices with dimensions of $4 k+2$, for $k \geq 1$.

\section{OFDM SYSTEMS WITH APU PRECODING MATRIX}

Linearly precoded OFDM systems have been studied by a number of researchers [20]-[22]. Of particular interest is the OFDM system with a DFT precoding matrix. Such a system was shown to be the same as the so-called single carrier with frequency domain equalizer (SC-FDE) system [23]. In [20], it was shown that the SC-FDE system has the maximum diversity gain among all linearly precoded OFDM systems. In [21], a bit error rate (BER) minimized precoder for an OFDM system was considered. For high SNR transmission, the SC-FDE system is optimal in the sense that it minimizes the bit error rate among OFDM systems with any orthogonal precoding matrix. In these studies, the precoders are constant matrices independent of $z$. In this section, we will study precoded OFDM systems with APU precoding matrices.

Fig. 3 shows the block diagram of a precoded OFDM system. In a precoded OFDM transmitter, the $k$ th input block $\mathbf{s}(k)$, consisting of $M$ modulation symbols such as QAM symbols, is first passed through an $M$ by $M$ precoding matrix $\mathbf{T}(z)$. The output of $\mathbf{T}(z)$ is given by

$$
\mathbf{u}(k)=\sum_{i=0}^{N-1} \mathbf{T}_{i} \mathbf{s}(k-i) .
$$

In this section, the precoding matrix $\mathbf{T}(z)$ is chosen as a normalized APU matrix so that

$$
\tilde{\mathbf{T}}(z) \mathbf{T}(z)=\mathbf{I}_{M}
$$

That means that all the entries are scaled by $1 \sqrt{M N}$. In practice, this normalization constant can be absorbed into the signal power of modulation symbols. After taking the $M$-point inverse 


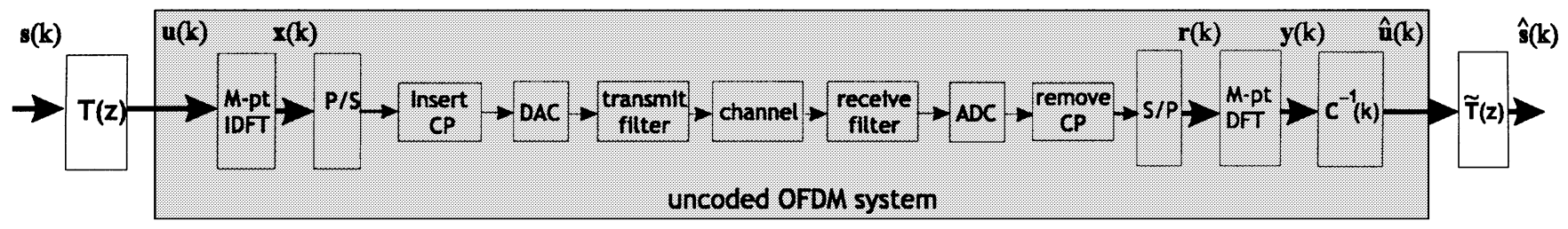

Fig. 3. OFDM system with APU precoding matrix $\mathbf{T}(z)$.

discrete Fourier transform (IDFT) of the vector $\mathbf{u}(k)$, we get the $M \times 1$ vector

$$
\mathbf{x}(k)=\mathbf{W}^{H} \mathbf{u}(k)
$$

where $\mathbf{W}$ is the DFT matrix with $[\mathbf{W}]_{k l}=1 / \sqrt{M} \exp$ $(-j 2 \pi k l / M)$. Note that unlike the conventional block transmission system, the transmitted block $\mathbf{x}(k)$ contains information of the input blocks $\mathbf{s}(k), \mathbf{s}(k-1), \ldots, \mathbf{s}(k-N+1)$. Before $\mathbf{x}(k)$ is transmitted, a cyclic prefix (CP) of length $L$ is added. In this paper, we assume that the channel is slowly varying so that for each OFDM block, the channel response does not vary. We model the combined effect of the digital-to-analog converter (DAC), transmit filter, channel, receive filter, and analog-to-digital converter (ADC) as an equivalent discrete time system with $c(n, k)$ denoting the $n$th tap of the impulse response when the $k$ th block is sent. We also assume that the CP length $L$ is large enough so that the length of the equivalent channel is $\leq(L+1)$, that is, for all $k, c(n, k)=0$ whenever $n>L+1$. The channel noise $\nu(n)$ is assumed to be additive white Gaussian noise (AWGN) with variance $N_{0}$. At the receiver end, to remove the interblock interference, the first $\mathrm{L}$ samples of the received block that correspond to the cyclic prefix are discarded. We obtain the $M \times 1$ vector $\mathbf{r}(k)$. Taking the DFT of $\mathbf{r}(k)$, we get

$$
\mathbf{y}(k)=\mathbf{W r}=\mathbf{C}(k) \mathbf{u}(k)+\boldsymbol{\nu}(k)
$$

where $\mathbf{C}(k)$ is an $M \times M$ diagonal matrix whose $(\ell, \ell)$ th entry is given by the DFT coefficient of the channel impulse response $c(n, k)$ :

$$
C_{\ell}(k)=\sum_{n=0}^{L+1} c(n, k) e^{-j 2 \pi n \ell / M} .
$$

The noise vector $\boldsymbol{\nu}(k)$ is an AWGN vector. Assume that the channel does not have spectral null so that $\mathbf{C}(k)$ is invertible. After multiplying the diagonal matrix $\mathbf{C}^{-1}(k)$, we get

$$
\hat{\mathbf{u}}(k)=\mathbf{u}(k)+\mathbf{C}^{-1}(k) \boldsymbol{\nu}(k) .
$$

In the absence of channel noise, the vector $\hat{\mathbf{u}}(k)=\mathbf{u}(k)$ for all $k$. When the precoding matrix $\mathbf{T}(z)$ is $\mathrm{PU}$, we can get a zero forcing receiver by taking $\tilde{\mathbf{T}}(z)$ as the decoding matrix, ${ }^{3}$ as indicated in Fig. 3. Note that when we take $\mathbf{T}(z)=\tilde{\mathbf{T}}(z)=$ $\mathbf{I}_{M}$, the system in Fig. 3 reduces to the conventional uncoded OFDM system. It should be emphasized that even though the

${ }^{3}$ For convenience, we use a noncausal decoding matrix. A causal receiver can be easily obtained by cascading enough delays. precoded OFDM system has an overlapping-block transmitter, the channel impulse response $c(n, k)$ can be different for different block indices $k$, and the system in Fig. 3 still has the zero-forcing property.

\section{A. Noise Analysis}

Define $\boldsymbol{\beta}(k)$ as

$$
\boldsymbol{\beta}(k)=\hat{\mathbf{u}}(k)-\mathbf{u}(k)=\mathbf{C}^{-1}(k) \boldsymbol{\nu}(k) .
$$

The autocorrelation matrices of $\boldsymbol{\beta}(k)$ are given by

$$
\mathcal{R}_{\beta}(k, \ell)=\mathcal{E}\left[\boldsymbol{\beta}(k) \boldsymbol{\beta}^{H}(k-\ell)\right]=N_{0} \delta(\ell) \mathbf{C}^{-1}(k) \mathbf{C}^{-H}(k)
$$

where $N_{0}$ is the variance of the channel noise $\nu(n)$. Because $\mathbf{C}^{-1}(k)$ are diagonal matrices, we see from the above equation that $\boldsymbol{\beta}(k)$ is also an AWGN vector, but each entry has a different variance.

Define the output noise vector $\mathbf{e}(k)=\hat{\mathbf{s}}(k)-\mathbf{s}(k)$. Then, it can be viewed as the output of $\tilde{\mathbf{T}}(z)$ with the input vector $\boldsymbol{\beta}(k)$. Therefore, we can write

$$
\mathbf{e}(k)=\sum_{\ell=0}^{N-1} \mathbf{T}_{\ell}^{H} \boldsymbol{\beta}(k+\ell) .
$$

Using the facts that $\boldsymbol{\beta}(k)$ is an AWGN vector and $\tilde{\mathbf{T}}(z)$ is a normalized PU matrix, one can verify that its zeroth autocorrelation matrix at the $k$ th block is given by

$$
\mathcal{R}_{e}(k, 0)=\mathcal{E}\left[\mathbf{e}(k) \mathbf{e}^{H}(k)\right]=\sum_{\ell=0}^{N-1} \mathbf{T}_{\ell}^{H} \mathcal{R}_{\beta}(k+\ell, 0) \mathbf{T}_{\ell}
$$

where $\mathcal{R}_{\beta}(i, 0)$ is the zeroth autocorrelation matrix of $\boldsymbol{\beta}(i)$ given in (28). Note that $\mathcal{R}_{\beta}(i, 0)$ is a diagonal matrix. Looking at the $i$ th diagonal term of $\mathcal{R}_{e}(k, 0)$, we can write the noise variance at $i$ th subband (when the $k$ th block is being processed) as

$$
\sigma_{i, \mathbf{T}}^{2}(k)=\frac{1}{N} \sum_{\ell=0}^{N-1}\left[\frac{1}{M} \sum_{n=0}^{M-1} \frac{N_{0}}{\left|C_{n}(k+\ell)\right|^{2}}\right]
$$

where we have used (28) and the fact that all the entries of $\mathbf{T}_{\ell}$ have magnitude equal to $1 / \sqrt{M N}$. The quantity $\sigma_{i, \mathbf{T}}^{2}(k)$ is independent of $i$; all subbands have the same noise variance! Moreover, the decoding matrix $\tilde{\mathbf{T}}(z)$ has an averaging effect on the channel gains over a time period of $N$ blocks. Note that we make no assumptions about the APU matrix $\mathbf{T}(z)$. Any APU precoding matrix can achieve (29). From (29), we also see that 


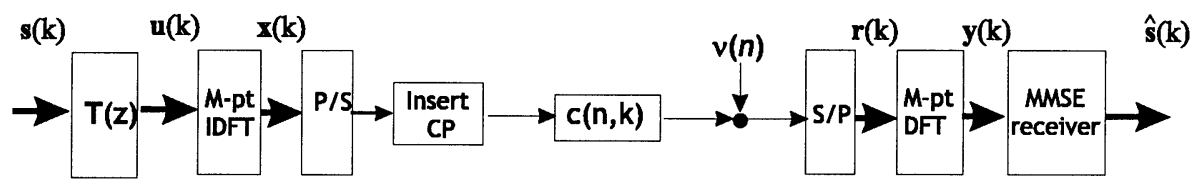

(a)

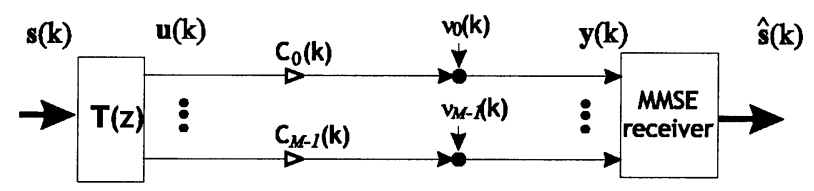

(b)

Fig. 4. (a) Precoded OFDM system with an MMSE receiver. (b) Equivalent system.

the performance of precoded OFDM with a zero-forcing receiver degrades significantly when one or some of the channel gains are small. The noise variances in all subbands will be very large over a period of $N$ blocks. To solve this problem, a minimum mean-square-error (MMSE) receiver is needed and will be derived in the next subsection.

1) Comparison With the Uncoded OFDM and SC-FDE Systems: When we take $\mathbf{T}(z)=\mathbf{I}_{M}$, the system in Fig. 3 becomes the conventional uncoded OFDM system. In this case, $\mathbf{u}(k)=\mathbf{s}(k)$. Thus, for an uncoded OFDM system, we can obtain from (27) the output noise variance at the $i$ th subband as

$$
\sigma_{i, \text { ofdm }}^{2}(k)=\frac{N_{0}}{\left|C_{i}(k)\right|^{2}} .
$$

The average output noise variance is

$$
\sigma_{\mathrm{av}, \mathrm{ofdm}}^{2}(k)=\frac{1}{M} \sum_{i=0}^{M-1} \frac{N_{0}}{\left|C_{i}(k)\right|^{2}} .
$$

From (30), we see that $\sigma_{i \text {,ofdm }}^{2}(k)$ depends on both $k$ and $i$, and it is inversely proportional to $\left|C_{i}(k)\right|^{2}$. Recall that $\left|C_{i}(k)\right|$ is the channel gain at the $i$ th frequency bin when the $k$ th block is sent. For highly frequency selective channels, some of the gains $\left|C_{i}(k)\right|$ can be close to zero, and the performance of the OFDM system will be affected by these spectral nulls.

If we allow the definition of APU matrices to include complex matrices whose coefficients have unit magnitude, then the DFT matrix $\mathbf{W}$ is APU. When we take $\mathbf{T}(z)=\mathbf{W}$, the system in Fig. 3 becomes the SC-FDE system [21]. By carrying out the same derivation, one can show that the noise variance of the SC-FDE system can be obtained by simply setting $N=1$ in (29). The noise variance at the $i$ th subband when the $k$ th block is sent is given by

$$
\sigma_{i, \mathrm{sc}}^{2}(k)=\frac{1}{M} \sum_{n=0}^{M-1} \frac{N_{0}}{\left|C_{n}(k)\right|^{2}} .
$$

Observe from the above expression that $\sigma_{i \text {,sc }}^{2}(k)$ is independent of $i$. All the subbands have the same noise variance, and they are equal to the average noise variance $\sigma_{\mathrm{av}, \mathrm{ofdm}}^{2}(k)$ in (31).

We can clearly see the difference between the conventional OFDM, the SC-FDE and the APU-precoded OFDM systems from the three expressions in (30), (32), and (29), respectively. Because the decoding matrices $\tilde{\mathbf{T}}(z)$ (for precoded OFDM system) and $\mathbf{W}^{H}$ (for SC-FDE system) are PU, they have the energy (or power) conservation property [1]. The average output noise variance for the three systems is the same. However, they distribute these noise variances to the subbands differently. For the conventional OFDM system, each subband can have a very different noise variance, especially when the channel is highly frequency selective. From (30), we see that subbands having small $\left|C_{i}(k)\right|$ will suffer from large noise variances. On the other hand, the SC-FDE system has an averaging effect in frequency domain; it averages over all subbands. When the channel has spectral nulls, all subbands will have large noise variances. To avoid this problem, an MMSE receiver is needed. Even when the channel does not have spectral nulls, for fast fading channel, the channel gains $C_{i}(k)$ can vary rapidly with respect to $k$. From (32), we see that if $\sum_{i}\left|C_{i}(k)\right|^{2}$ is small for some $k$, the whole $k$ th block will be severely affected by noise amplification problem. The APU-precoded OFDM system has an averaging effect in both the frequency and time domains; it averages over all subbands and over $N$ OFDM blocks. Similarly, if the channel has spectral nulls, all the subbands will have large noise variances for the next $N$ transmission blocks. Hence, an MMSE receiver is needed for the OFDM system with APU precoding matrices.

\section{B. MMSE Receiver for Precoded OFDM Systems}

We assume that the receiver removes the first $L$ samples corresponding to the cyclic prefix so that there is no interblock interference. Given the received vector $\mathbf{r}(k)$, we want to design an MMSE receiver. As the DFT matrix $\mathbf{W}$ is invertible, there is no loss of generality if we consider the system in Fig. 4(a). It is known that when the length of the channel length is $\leq L+1$, the frequency-selective channel $c(n, k)$ is converted into a set of $M$ parallel nonfrequency-selective subchannels. We can redraw the system in Fig. 4(a) as Fig. 4(b), where $\mathbf{C}(k)$ is a diagonal matrix whose $(\ell, \ell)$ th entry is the $\ell$ th DFT coefficient of $c(n, k)$, as defined in (26). The equivalent noise is the AWGN vector $\boldsymbol{\nu}(k)$ with the power spectral matrix $N_{0} \mathbf{I}_{M}$. In the following derivations, we assume that the transmitted signals $\mathbf{s}(k)$ satisfy

$$
\mathcal{E}\left\{\mathbf{s}(k) \mathbf{s}^{H}(k-\ell)\right\}=E_{s} \delta(\ell) \mathbf{I}_{M}
$$


In other words, the symbols are uncorrelated and have equal signal power. The fact that $\mathbf{T}(z)$ is normalized PU implies that $\mathbf{u}(k)$ also satisfies

$$
\mathcal{E}\left\{\mathbf{u}(k) \mathbf{u}^{H}(k-\ell)\right\}=E_{s} \delta(\ell) \mathbf{I}_{M}
$$

Moreover, we also assume that the transmitted signals are uncorrelated to the channel noise.

Consider an MMSE receiver (possibly time-varying) with $N$ coefficient matrices $\mathbf{Q}(k, \ell)$ for $0 \leq \ell \leq N-1$. Given the input vector $\mathbf{y}(k)$, the output of the MMSE receiver can be described as

$$
\hat{\mathbf{s}}(k)=\sum_{\ell=0}^{N-1} \mathbf{Q}(k, \ell) \mathbf{y}(k+\ell)
$$

where $\mathbf{Q}(k, \ell)$ are $M \times M$ matrices. For convenience, we consider the noncausal system. Our goal is to find $\mathbf{Q}(k, \ell)$ so that the following mean square error is minimized:

$$
\mathcal{E}\left\{(\hat{\mathbf{s}}(k)-\mathbf{s}(k))^{H}(\hat{\mathbf{s}}(k)-\mathbf{s}(k))\right\} .
$$

Applying the orthogonality principle, one can show that the MMSE solution is given by (see the Appendix for a proof)

$$
\mathbf{Q}(k, \ell)=\mathbf{T}_{\ell}^{H} \boldsymbol{\Lambda}(k+\ell)
$$

where $\boldsymbol{\Lambda}(i)=E_{s} \mathbf{C}^{H}(i)\left(E_{s} \mathbf{C}(i) \mathbf{C}^{H}(i)+N_{0} \mathbf{I}_{M}\right)^{-1}$. Note that $\boldsymbol{\Lambda}(k)$ is a diagonal matrix whose $n$th diagonal entry is given by

$$
\lambda_{n}(k)=\frac{C_{n}^{*}(k)}{\left|C_{n}(k)\right|^{2}+N_{0} / E_{s}} .
$$

From the above expressions, we see that the MMSE receiver can be decomposed into a time-varying diagonal matrix $\boldsymbol{\Lambda}(k)$ and the time-invariant matrix $\tilde{\mathbf{T}}(z)$. Therefore, we can implement the MMSE receiver as Fig. 5. Comparing the zero-forcing and MMSE receivers in Figs. 3 and 5, respectively, one immediately sees that their only difference is the one-tap equalizer. When there is no noise, i.e., $N_{0}=0$, the MMSE receiver reduces to the zero-forcing receiver.

One can verify that for the precoded OFDM system with an MMSE receiver, all the subbands also have the same error variance, and it is given by

$$
\sigma_{\mathrm{mmse}}^{2}(k)=\frac{1}{M N} \sum_{\ell=0}^{N-1} \sum_{n=0}^{M-1} \underbrace{\frac{N_{0}}{\left|C_{n}(k+\ell)\right|^{2}+N_{0} / E_{s}}}_{\sigma^{2}(n, \ell, k)} .
$$

One can clearly see from the above expression that the decoding matrix $\tilde{\mathbf{T}}(z)$ has an averaging effect in both frequency and time domains; it averages over all subbands and over $N$ OFDM blocks. Moreover, the quantities $\sigma^{2}(n, \ell, k)$ are upper bounded by $E_{s}$. When some of the channel gains $\left|C_{n}(k+\ell)\right|$ approach zero, the error variance $\sigma_{\mathrm{mmse}}^{2}(k)$ does not go to infinity. We will see in the next section that by using an MMSE receiver,

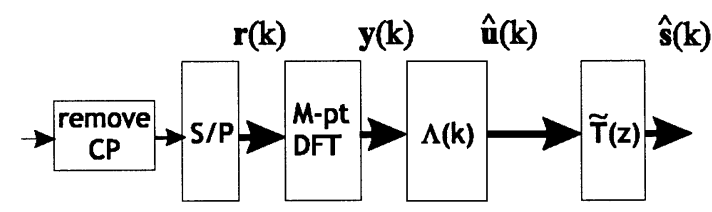

Fig. 5. Implementation of the MMSE receiver.

the performance of the precoded OFDM system is improved significantly.

1) Precoders With Missing Powers in z: From (37), we see that when the channel gains $C_{n}(i)$ are slow-varying and remain virtually the same for $N$ blocks, then averaging effect becomes negligible, as we will see in the next section. One can increase the length of the precoder so that more terms are averaged, but this will increase the computational complexity. To avoid this, one can perform averaging over nonconservative blocks. To do this, we can use precoders with missing powers in $z$. Let $\mathbf{T}(z)$ be an APU matrix. Then, $\mathbf{T}\left(z^{J}\right)$ for integer $J$ is also APU. Using $\mathbf{T}\left(z^{J}\right)$ as a precoder, the output error variance of all subbands becomes

$$
\sigma_{\mathrm{mmse}}^{2}(k)=\frac{1}{M N} \sum_{\ell=0}^{N-1} \sum_{n=0}^{M-1} \frac{N_{0}}{\left|C_{n}(k+\ell J)\right|^{2}+N_{0} / E_{s}} .
$$

Compared with $\mathbf{T}(z)$, we need only extra delays to implement $\mathbf{T}\left(z^{J}\right)$, and the computational complexity remains the same.

2) Choice of APU Matrices: As we have mentioned above, given the length and dimension of the precoding matrix, all APU matrices have the same noise property. In practice, one can consider other criteria when choosing the APU matrices. For example, one can consider the peak-to-average power ratio (PAPR). It is well-known that OFDM system suffers from high PAPR. The PAPR of an $M$-channel OFDM system is proportional to $\sqrt{M}$. For OFDM systems with an APU precoding matrix, one can choose the APU matrix to alleviate the PAPR problem. To see this, we can use a complex APU matrix of the form

$$
\mathbf{T}(z)=\mathbf{W} \boldsymbol{\theta}\left(z^{0}\right) \mathcal{B} \boldsymbol{\theta}\left(z^{2^{1}}\right) \mathcal{B} \ldots \boldsymbol{\theta}\left(z^{2^{J}}\right) \mathcal{B}
$$

where $\mathbf{W}$ is the $M$ by $M$ DFT matrix, and $\boldsymbol{\theta}(z)$ and $\mathcal{B}$ are the $M$ by $M$ matrices defined in (15). The length of $\mathbf{T}(z)$ is $N=2^{J+1}$. Consider Fig. 3. Because $\mathbf{W}^{H} \mathbf{W}=\mathbf{I}$, the transfer matrix from the input vector $\mathbf{s}(k)$, consisting of transmitted symbols, to $\mathbf{x}(k)$ is

$$
\boldsymbol{\theta}\left(z^{0}\right) \mathcal{B} \boldsymbol{\theta}\left(z^{2^{1}}\right) \mathcal{B} \ldots \boldsymbol{\theta}\left(z^{2^{J}}\right) \mathcal{B} .
$$

Note that there are only $(J+1)$ stages of butterfly. The PAPR is at most $\sqrt{2}$ for each stage. Hence, the PAPR is at most $(\sqrt{2})^{J+1}$, which is equal to $\sqrt{N}$. In practice, $N$ is usually much smaller than $M$. One additional advantage of using the APU matrix $\mathbf{T}(z)$ of the form in (38) is that there is no need to implement both the IDFT matrix $\mathbf{W}^{H}$ and the DFT matrix $\mathbf{W}$ at the transmitter. This leads to an additional computational saving. Another possible criterion that can be incorporated into the design 


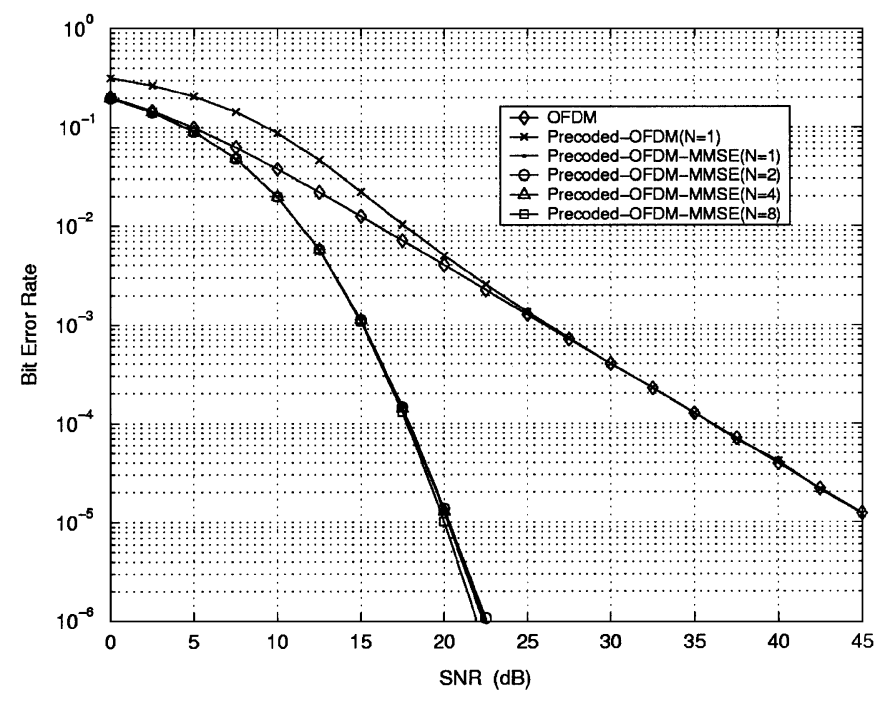

Fig. 6. Bit error rate performance for slowly varying channels.

of APU matrices is the frequency response of the transmit filters. This problem is beyond the scope of this paper.

\section{SimULATION}

In this section, we carry out Monte Carlo experiments to verify the performance of precoded OFDM systems with different precoders. The transmission channels are the modified Jakes fading channels described in [24]. In the experiments, we will use channel models with two different ratios of doppler frequency over transmission bandwidth. A larger value of $r$ indicates that the channel is changing faster. The ratio $r=0.0001$ corresponds to a slowly varying channel, whereas $r=0.001$ corresponds to a channel that varies ten times faster. The number of taps of the channels is 16 . The channel noise $\nu(n)$ is AWGN with variance $N_{0}$. In our simulation, we assume that the receiver knows the exact channel response. The DFT size is $M=64$, and the length of cyclic prefix is $L=16$. The input vector $\mathbf{s}(n)$ consists of quadrature phase shift keying (QPSK) symbols with power equal to $E_{s}$.

APU matrices of different length $N$ will be used as the precoding matrices. When $N=1$, the APU matrix reduces to the Hadamard matrix. It is known [21] that the OFDM system with a Hadamard precoding matrix has the same BER performance as the SC-FDE system. We plot the BER curves versus the signal-to-noise ratio (SNR), which is equal to $E_{s} / N_{0}$. In the simulation, we do not consider the MMSE receiver for the conventional OFDM system because the BER performance of OFDM systems with MMSE receivers is identical to that of OFDM systems with zero-forcing receivers.

The results for $r=0.0001$ are shown in Fig. 6. From the figure, we see that the performance of precoded OFDM system with a zero-forcing receiver is worse than that of the OFDM system at low SNR. This is because when the transmission encounters deep fading at some frequency bins, all the outputs of precoded OFDM receiver will be seriously affected by channel noise. On the other hand, for the OFDM system, only a portion of the outputs will be seriously affected. However, when an MMSE receiver is employed, the precoded OFDM systems

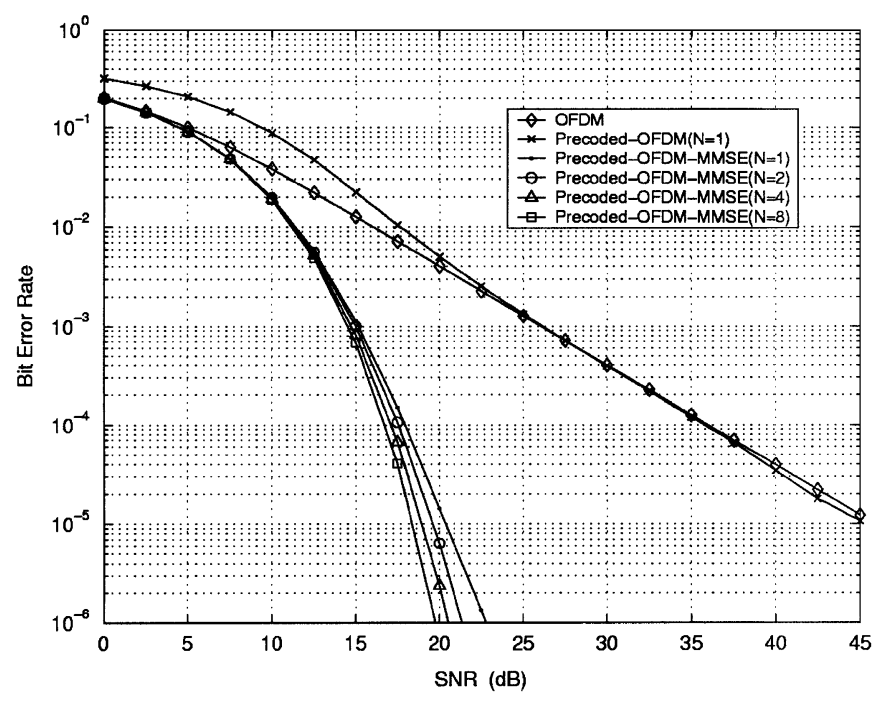

Fig. 7. Bit error rate performance for fast varying channels.

have a much better performance than the OFDM system. If we compare the performance of precoded OFDM systems with different precoders, we see that when the channel is slowly varying, using a longer precoding matrix does not provide much gain in performance. This is because when the channel variation in the time domain is small, averaging the performance in the time domain has little effect on the performance.

For channel that is varying ten times faster with $r=0.001$, the results are shown in Fig. 7. Again, we see that the precoded OFDM system with a zero-forcing receiver does not perform well, and using an MMSE receiver can greatly improve the performance of precoded OFDM systems. In addition, note that the performance improves as the length of the precoding matrix $N$ increases. As the channel is fast varying, averaging in the time domain can provide additional gain. If we compare the cases of $N=1$ and $N=8$, averaging over eight blocks can yield an additional gain of more than $2 \mathrm{~dB}$ when the BER is $10^{-5}$.

\section{CONCLUSION}

In this paper, a $z$-domain approach is introduced to study APU matrices. This approach not only gives a compact description of the previous results but also enables us to generalize previous methods. Moreover, several new methods for the construction of APU matrices have been introduced. In particular, APU matrices constructed by the butterfly method have the additional advantage of low implementational cost. We have also considered application of APU matrices in precoded OFDM systems. We have showed that by using an APU precoding matrix, the output noise is averaged in both time and frequency domain. Simulation results show that precoded OFDM systems with MMSE receivers have a significantly better performance than the conventional OFDM system.

\section{APPENDIX}

\section{A. Proof of Generalized AS Method}

In the following, we will show that the matrix $\mathbf{C}(z)=$ $\mathbf{A}\left(z^{N_{b}}\right) \otimes_{A S} \mathbf{B}(z)$ is APU. Recall that the matrix $\mathbf{C}(z)$ 
is given in (14). Define the two matrices $\mathbf{P}_{i}(z)=$ $(1 / 2)\left(\mathbf{A}_{i 0}(z)+\mathbf{A}_{i 1}(z)\right)$ and $\mathbf{Q}_{i}(z)=(1 / 2)\left(\mathbf{A}_{i 0}(z)-\mathbf{A}_{i 1}(z)\right)$. Let $\mathbf{P}_{i}(z)=\sum_{n} \mathbf{p}_{i}(n) z^{-n}$ and $\mathbf{Q}_{i}(z)=\sum_{n} \mathbf{q}_{i}(n) z^{-n}$. Then, one can verify that the entries of the coefficient matrices $\mathbf{p}_{i}(n)$ and $\mathbf{q}_{i}(n)$ consist of either \pm 1 or 0 . Moreover, if $\left[\mathbf{p}_{i}(n)\right]_{k l}= \pm 1$, then $\left[\mathbf{q}_{i}(n)\right]_{k l}=0$ and vice versa. Using this result, one can see from (14) that $\mathbf{C}(z)$ is antipodal. To show that $\mathbf{C}(z)$ is PU, we need to prove that $\mathbf{C}(z) \mathbf{C}(z)=\alpha \mathbf{I}_{M_{a} M_{b} / 2}$ for some positive constant $\alpha$. In the following, we will show that

$$
\tilde{\mathbf{C}}_{00}(z) \mathbf{C}_{00}(z)+\tilde{\mathbf{C}}_{10}(z) \mathbf{C}_{10}(z)=\alpha \mathbf{I}_{M_{a} M_{b} / 4}
$$

The proof for other terms is very similar. For notational complexity, we will drop the dependency on $z$. Using the fact that

$$
(\mathbf{A}(z) \otimes \mathbf{B}(z))^{\sim}=\tilde{\mathbf{A}}(z) \otimes \tilde{\mathbf{B}}(z),
$$

we can write

$$
\begin{aligned}
4\left(\tilde{\mathbf{C}}_{00} \mathbf{C}_{00}+\tilde{\mathbf{C}}_{10} \mathbf{C}_{10}\right) & \left.\left.\tilde{\mathbf{A}}_{00}+\tilde{\mathbf{A}}_{01}\right) \otimes \tilde{\mathbf{B}}_{00}+\left(\tilde{\mathbf{A}}_{00}-\tilde{\mathbf{A}}_{01}\right) \otimes \tilde{\mathbf{B}}_{10}\right] \\
= & {\left[\left(\tilde{\mathbf{A}}_{00}-\mathbf{A}_{01}\right) \otimes \mathbf{B}_{10}\right] } \\
& \times\left[\left(\mathbf{A}_{00}+\mathbf{A}_{01}\right) \otimes \mathbf{B}_{00}+\left(\mathbf{A}_{00}-\tilde{\mathbf{A}}_{11}\right) \otimes \tilde{\mathbf{B}}_{10}\right] \\
& +\left[\left(\tilde{\mathbf{A}}_{10}+\tilde{\mathbf{A}}_{11}\right) \otimes \tilde{\mathbf{B}}_{00}+\left(\tilde{\mathbf{A}}_{10}-\mathbf{A}_{11}\right) \otimes \mathbf{B}_{10}\right] . \\
& \times\left[\left(\mathbf{A}_{10}+\mathbf{A}_{11}\right) \otimes \mathbf{B}_{00}+\left(\mathbf{A}_{10}-\mathbf{A}_{10}\right.\right.
\end{aligned}
$$

Using the product rule (1), one can write

$$
\begin{aligned}
4\left(\tilde{\mathbf{C}}_{00}\right. & \left.\mathbf{C}_{00}+\tilde{\mathbf{C}}_{10} \mathbf{C}_{10}\right) \\
= & {\left[\left(\tilde{\mathbf{A}}_{00}+\tilde{\mathbf{A}}_{01}\right)\left(\mathbf{A}_{00}+\mathbf{A}_{01}\right)+\left(\tilde{\mathbf{A}}_{10}+\tilde{\mathbf{A}}_{11}\right)\right.} \\
& \left.\times\left(\mathbf{A}_{10}+\mathbf{A}_{11}\right)\right] \otimes \tilde{\mathbf{B}}_{00} \mathbf{B}_{00} \\
& +\left[\left(\tilde{\mathbf{A}}_{00}-\tilde{\mathbf{A}}_{01}\right)\left(\mathbf{A}_{00}-\mathbf{A}_{01}\right)+\left(\tilde{\mathbf{A}}_{10}-\tilde{\mathbf{A}}_{11}\right)\right. \\
& \left.\times\left(\mathbf{A}_{10}-\mathbf{A}_{11}\right)\right] \otimes \tilde{\mathbf{B}}_{10} \mathbf{B}_{10} \\
& +\left[\left(\tilde{\mathbf{A}}_{00}+\tilde{\mathbf{A}}_{01}\right)\left(\mathbf{A}_{00}-\mathbf{A}_{01}\right)+\left(\tilde{\mathbf{A}}_{10}+\tilde{\mathbf{A}}_{11}\right)\right. \\
& \left.\times\left(\mathbf{A}_{10}-\mathbf{A}_{11}\right)\right] \otimes \tilde{\mathbf{B}}_{00} \mathbf{B}_{10} \\
& +\left[\left(\tilde{\mathbf{A}}_{00}-\tilde{\mathbf{A}}_{01}\right)\left(\mathbf{A}_{00}+\mathbf{A}_{01}\right)+\left(\tilde{\mathbf{A}}_{10}-\tilde{\mathbf{A}}_{11}\right)\right. \\
& \left.\times\left(\mathbf{A}_{10}+\mathbf{A}_{11}\right)\right] \otimes \tilde{\mathbf{B}}_{10} \mathbf{B}_{00} .
\end{aligned}
$$

Using the fact that $\tilde{\mathbf{A}} \mathbf{A}=\alpha_{a} \mathbf{I}_{M_{a}}$ for some positive $\alpha_{a}$, we can simplify the above equation as

$$
\begin{aligned}
4\left(\tilde{\mathbf{C}}_{00} \mathbf{C}_{00}+\right. & \left.\tilde{\mathbf{C}}_{10} \mathbf{C}_{10}\right) \\
& =2 \alpha_{a} \mathbf{I}_{M_{a} / 2} \otimes \tilde{\mathbf{B}}_{00} \mathbf{B}_{00}+2 \alpha_{a} \mathbf{I}_{M_{a} / 2} \otimes \tilde{\mathbf{B}}_{10} \mathbf{B}_{10}
\end{aligned}
$$

Because $\tilde{\mathbf{B} B}=\alpha_{b} \mathbf{I}_{M_{b}}$ for some positive $\alpha_{b}$, we can write $4\left(\tilde{\mathbf{C}}_{00} \mathbf{C}_{00}+\tilde{\mathbf{C}}_{10} \mathbf{C}_{10}\right)=4 \alpha_{a} \alpha_{b} \mathbf{I}_{M_{a} M_{b} / 4}$, which proves the result.

\section{B. Derivation of the MMSE Receiver}

Using the orthogonality principle, the MMSE receiver should satisfy

$$
\mathcal{E}\left\{(\hat{\mathbf{s}}(k)-\mathbf{s}(k)) \mathbf{y}^{H}(k+\ell)\right\}=0, \quad \text { for } 0 \leq l<N .
$$

From (25), (27), and (33), we immediately get

$$
\mathcal{E}\left\{\mathbf{s}(k) \mathbf{y}^{H}(k+\ell)\right\}=E_{s} \mathbf{T}_{\ell}^{H} \mathbf{C}^{H}(k+\ell) .
$$

Using (34), one can show that

$$
\begin{array}{r}
\mathbf{y}(k+m) \mathbf{y}^{H}(k+\ell) \\
\quad=\left(N_{0} \mathbf{I}_{M}+E_{s} \mathbf{C}(k+\ell) \mathbf{C}^{H}(k+\ell)\right) \delta(m-\ell) .
\end{array}
$$

Using the above result and (35), we have

$$
\begin{aligned}
\mathcal{E}\left\{\hat{\mathbf{s}}(k) \mathbf{y}^{H}(k+\ell)\right\} & \\
& =\mathbf{Q}(k, \ell)\left(N_{0} \mathbf{I}_{M}+E_{s} \mathbf{C}(k+\ell) \mathbf{C}^{H}(k+\ell)\right) .
\end{aligned}
$$

Substituting (40) and (41) into (39), we immediately get the result in (36).

\section{ACKNOWLEDGMENT}

The authors would like to thank Prof. S. C. Pei, National Taiwan University, Taipei, for bringing our attention to the results on Hadamard matrices with non power-of-two dimensions. They would also like to thank the anonymous reviewers and Prof. P. P. Vaidyanathan, Caltech, Pasadena, CA, for their constructive suggestions, which have significantly improved the manuscript.

\section{REFERENCES}

[1] P. P. Vaidyanathan, Multirate Systems and Filterbanks. Englewood Cliffs, NJ: Prentice-Hall, 1993.

[2] M. Vetterli and J. Kovacevic, Wavelets and Subband Coding. Englewood Cliffs, NJ: Prentice-Hall, 1995.

[3] G. Strang and T. Nguyen, Wavelets and Filterbanks. Wellesley, MA: Wellesley-Cambridge Press, 1996.

[4] M. J. T. Smith and T. P. Barnwell III, "A procedure for designing exact reconstruction filterbanks for tree-structured subband coders," in Proc. IEEE Int. Conf. Acoust. Speech, Signal Process., San Diego, CA, Mar. 1984, pp. 27.1.1-27.1.4.

[5] P. P. Vaidyanathan, "Theory and design of $M$-channel maximally decimated quadraature mirror filters with arbitrary $M$, having perfect reconstruction property," IEEE Trans. Acoust., Speech, Signal Process., vol. ASSP-35, pp. 476-492, Apr. 1987.

[6] M. J. E. Golay, “Complementary series," IRE Trans. Inf. Theory, vol. IT-7, pp. 82-87, 1961.

[7] Y. Taki, H. Miyakawa, M. Hatori, and S. Namba, "Even-shift orthogonal sequences," IEEE Trans. Inf. Theory, vol. IT-15, pp. 295-300, Mar. 1969.

[8] J. Seberry and M. Yamada, "Hadamard matrices, sequences, and block designs," in Contemporary Design Theory: A Collection of Surveys, J. H. Dinitz and D. R. Stinson, Eds. New York: Wiley, 1992.

[9] C.-C. Tseng and C. L. Liu, "Complementary sets of sequences," IEEE Trans. Inf. Theory, vol. IT-18, pp. 644-652, Sep. 1972.

[10] N. Suehiro and M. Hatori, "N-shift cross-orthogonal sequences," IEEE Trans. Inf. Theory, vol. 34, no. 1, pp. 143-146, Jan. 1988. 
[11] G. W. Wornell, "Spread signature CDMA: Efficient multiuser communication in the presence of fading," IEEE Trans. Inf. Theory, vol. 41, no. 5, pp. 1418-1438, Sep. 1995.

[12] H. Chen, J. Yeh, and N. Suehiro, "A multicarrier CDMA architecture based on orthogonal complementary codes for new generations of wideband wireless communications," IEEE Commun. Mag., vol. 39, no. 10, pp. 126-135, Oct. 2001

[13] H. S. Shapiro, "Extremal Problems for Polynomials and Power Series," Master's Thesis, Mass. Inst. Technol., Cambridge, MA, 1951.

[14] W. Rudin, "Some theorems on Fourier coefficients," in Proc. Amer. Math. Soc., vol. 10, 1959, pp. 855-859.

[15] S. M. Phoong and Y. P. Lin, "Lapped Hadamard transforms and filterbanks," in Proc. Int. Conf. Acoust. Speech, Signal Processing, Apr. 2003.

[16] S. M. Phoong, K. Y. Chang, and Y. P. Lin, "Antipodal paraunitary precoding for OFDM application," in Proc. Int. Symp. Circuits Systems, May 2004.

[17] R. E. A. C. Paley, "On orthogonal matrices," J. Math. Phys., vol. 12, pp. 311-320, 1933

[18] S. M. Phoong and P. P. Vaidyanathan, "Paraunitary filter banks over finite fields," IEEE Trans. Signal Processing, vol. 45, no. 6, pp. 1443-1457, Jun. 1997.

[19] N. Ahmed and K. R. Rao, Orthogonal Transforms for Digital Signal Processing. New York: Springer-Verlag, 1975.

[20] Z. Wang and G. B. Giannakis, "Linearly precoded or coded OFDM against wireless channel fades?," in Proc. Third IEEE Workshop Signal Process. Adv. Wireless Commun., Taoyuan, Taiwan, R.O.C., Mar. 2001.

[21] Y. P. Lin and S. M. Phoong, "BER minimized OFDM systems with channel independent precoders," IEEE Trans. Signal Process., vol. 51, no. 9, pp. 2369-2380, Sept. 2003.

[22] Y. Ding, T. N. Davidson, Z.-Q. Luo, and K. M. Wong, "Minimum BER block precoders for zero-forcing equalization," IEEE Trans. Signal Process., vol. 51, no. 9, pp. 2410-2423, Sep. 2003.

[23] H. Sari, G. Karam, and I. Jeanclaude, Frequency-Domain Equalization of Mobile Radio and Terrestrial Broadcast Channels. San Francisco, CA: Globecom, 1994.

[24] P. Dent, G. E. Bottomley, and T. Croft, "Jakes fading model revisited," Electron. Lett., pp. 1162-1163, Jun. 1993.

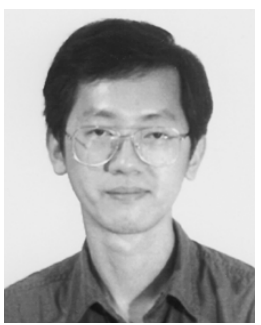

See-May Phoong (M'96-SM'04) was born in Johor, Malaysia, in 1968. He received the B.S. degree in electrical engineering from the National Taiwan University (NTU), Taipei, Taiwan, R.O.C., in 1991 and the M.S. and Ph.D. degrees in electrical engineering from the California Institute of Technology (Caltech), Pasadena, in 1992 and 1996, respectively.

He was with the Faculty of the Department of Electronic and Electrical Engineering, Nanyang Technological University, Singapore, from September 1996 to September 1997. In September 1997, he joined the Graduate Institute of Communication Engineering and the Department of Electrical Engineering, NTU, as an Assistant Professor, and since August 2001, he has been an Associate Professor.

Dr. Phoong is currently an Associate Editor for the IEEE SIGNAL PROCESSING LETTERS. He has previously served as an Associate Editor for the IEEE TRANSACtions on Circuits and Systems II: ANAlog AND Digital Signal PROCESSING from January 2002 to December 2003. His interests include multirate signal processing, filterbanks, and their application to communications.

Dr. Phoong received the Charles $\mathrm{H}$. Wilts Prize for outstanding independent research in electrical engineering at Caltech in 1997.

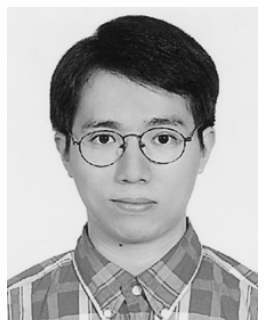

Kaiyen Chang was born in Taipei, Taiwan, R.O.C., in 1977. He received the B.S. degree in electrical engineering from the National Tsing Hua University (NTHU), Hsinchu, Taiwan, in 1999 and the M.S. degree in communication engineering from the National Taiwan University, Taipei, Taiwan, in 2003.

His research focuses on the orthogonal frequency division multiplex systems. 